Article

\title{
An Insight into the Structural Requirements and Pharmacophore Identification of Carbonic Anhydrase Inhibitors to Combat Oxidative Stress at High Altitudes: An In-Silico Approach
}

\author{
Amena Ali 1,2,*(D), Abuzer Ali ${ }^{3}{ }^{(D)}$, Musarrat Husain Warsi ${ }^{4}$, Mohammad Akhlaquer Rahman ${ }^{4}(\mathbb{D}$, \\ Mohamed Jawed Ahsan ${ }^{5}$ (D) and Faizul Azam ${ }^{6}$ (D)
}

check for

updates

Citation: Ali, A.; Ali, A.; Warsi, M.H.; Rahman, M.A.; Ahsan, M.J.; Azam, F. An Insight into the Structural Requirements and Pharmacophore Identification of Carbonic Anhydrase Inhibitors to Combat Oxidative Stress at High Altitudes: An In-Silico Approach. Curr. Issues Mol. Biol. 2022, 44, 1027-1045. https://doi.org/ 10.3390/cimb44030068

Academic Editor: Hidayat Hussain

Received: 11 January 2022

Accepted: 16 February 2022

Published: 23 February 2022

Publisher's Note: MDPI stays neutral with regard to jurisdictional claims in published maps and institutional affiliations.

Copyright: (C) 2022 by the authors. Licensee MDPI, Basel, Switzerland. This article is an open access article distributed under the terms and conditions of the Creative Commons Attribution (CC BY) license (https:// creativecommons.org/licenses/by/ $4.0 /)$.
1 High Altitude Research Center, Taif University, P.O. Box 11099, Taif 21944, Saudi Arabia

2 Department of Pharmaceutical Chemistry, College of Pharmacy, Taif University, P.O. Box 11099, Taif 21944, Saudi Arabia

3 Department of Pharmacognosy, College of Pharmacy, Taif University, P.O. Box 11099, Taif 21944, Saudi Arabia; abuali@tu.edu.sa

4 Department of Pharmaceutics and Industrial Pharmacy, College of Pharmacy, Taif University, P.O. Box 11099, Taif 21944, Saudi Arabia; mvarsi@tu.edu.sa (M.H.W.); mrahman@tu.edu.sa (M.A.R.)

5 Department of Pharmaceutical Chemistry, Maharishi Arvind College of Pharmacy, Ambabari Circle, Jaipur 302 039, Rajasthan, India; jawedpharma@gmail.com

6 Department of Pharmaceutical Chemistry \& Pharmacognosy, Unaizah College of Pharmacy, Qassim University, P.O. Box 5888, Unaizah 51911, Saudi Arabia; f.azam@qu.edu.sa

* Correspondence: amrathore@tu.edu.sa

Abstract: Carbonic anhydrases (CA) inhibitory action could be linked to the treatment of a number of ailments, including cancer, osteoporosis, glaucoma, and several neurological problems. For the development of effective CA inhibitors, a variety of heterocyclic rings have been investigated. Furthermore, at high altitudes, oxygen pressure drops, resulting in the formation of reactive oxygen and nitrogen species, and CA inhibitors having role in combating this oxidative stress. Acetazolamide contains thiadiazole ring, which has aroused researchers' interest because of its CA inhibitory action. In the present study, we used a number of drug design tools, such as pharmacophore modeling, 3D QSAR, docking, and virtual screening on twenty-seven 1,3,4-thiadiazole derivatives that have been described as potential CA inhibitors in the literature. An atom-based 3D-QSAR analysis was carried out to determine the contribution of individual atoms to model generation, while a pharmacophore mapping investigation was carried out to find the common unique pharmacophoric properties required for biological activity. The coefficient of determination for both the training and test sets were statistically significant in the generated model. The best QSAR model was chosen based on the values of $R^{2}(0.8757)$ and $Q^{2}(0.7888)$. A molecular docking study was also conducted against the most potent analogue $4 \mathrm{~m}$, which has the highest SP docking score (-5.217) (PDB ID: 6g3v). The virtual screening revealed a number of promising compounds. The screened compound ZINC77699643 interacted with the amino acid residues, Pro201 and Thr199, in the virtual screening study (PDB ID: $6 \mathrm{~g} 3 \mathrm{v})$. These interactions demonstrated the significance of the CA inhibitory activity of the compound. Furthermore, ADME study revealed useful information regarding compound's drug-like properties. Therefore, the findings of the present investigation could aid in the development of more potent CA inhibitors, which could benefit the treatment of oxidative stress at high altitudes.

Keywords: 1,3,4-thiadiazole; oxidative stress; molecular modeling; carbonic anhydrase (CA) inhibitor; high altitude

\section{Introduction}

At high altitudes, oxygen pressure drops, resulting in the formation of reactive oxygen and nitrogen species (RONS), which have been linked to a variety of oxidative stress 
related diseases [1,2]. The formation of reactive oxygen and nitrogen species (RONS) causes oxidative damage to biomolecules such as protein and DNA, which has been linked to a number of diseases [3-6]. At high altitude, the body's antioxidant enzymes system becomes unresponsive. In addition, at this height, the RONS-producing sources, which include various mitochondrial transport systems, nitric oxide synthase, and xanthine oxidase, become activated [7-10]. Physical activity can sometimes cause an increase in oxidative stress, fatigue, headaches, nausea, anorexia, and poor sleep. A number of medications are available to treat oxidative stress, including carbonic anhydrase (CA) inhibitors [11] and, in particular, acetazolamide [12]. The literature survey revealed that acetazolamide (a specific inhibitor of CA) is used to treat high-altitude pulmonary oedema (HAPE) [13].

Carbonic anhydrase enzyme of various types is found in prokaryotes and eukaryotes. Carbonic anhydrases (CAs, EC 4.2.1.1) encode bacteria found in three different genetic families, the $\alpha_{-}, \beta-$, and $\gamma$-classes. These metalloenzymes interfere with $\mathrm{pH}$ regulation and other important physiological processes in these organisms by equilibrating $\mathrm{CO} 2$ and bicarbonate. These enzymes differ in terms of tissue expression, kinetic properties, and localization in different parts of the body. CAs I, II, III, VII, and XIII are cytosolic carbonic enzymes, whereas CAs IV, IX, XII, and XIV are cell membrane associated enzymes [14]. CAs VA and VB are found near the mitochondrial region, while VI is found in saliva and milk. Catalytic activity is absent in CAs VIII, X, and XI. CAs play various roles in our bodies, including electrolyte balance in various organs, biosynthetic reactions, calcification, bone resorption, $\mathrm{pH}$ and $\mathrm{CO}_{2}$ homeostasis, and tumorigenicity.

CAs' inhibitory activity has been linked to the treatment of a number of diseases, including cancer, osteoporosis, glaucoma, and some neurological disorders [15]. The use of heterocyclic rings in the development of powerful CA inhibitors has been studied. The thiadiazole (Acetazolamide) ring has piqued scientists' interest due to its ability to inhibit a variety of diseases [16,17].

In the current study, we used 3D QSAR to identify the structural requirements of 1,3,4-thiadiazole derivatives for potential human CA-I inhibitions. The presence of various pharmacophoric features such as aromatic groups, electron donating groups, electron withdrawing groups, and hydrophobic long chains was used to generate pharmacophore models, which are important for their inhibitory activities against CA. Furthermore, the association of CA inhibitors with the binding pocket of receptor was validated by a comparative molecular docking investigation, confirming their inhibitory efficacy. The pharmacophore predicted by the 3D QSAR analysis could be a viable scaffold for the identification of new CA inhibitors as anticancer agents. The virtual screening investigation was carried out using the ZINC database's pharmacophore [18]. Earlier findings suggested that acetazolamide drug can be taken as reference to produce different hypothetical compounds followed by their docking studies against CA enzyme target. After that, the best compounds were chosen by combining it with ZINC-screened compounds [19]. The hypothetic compounds can also be used for synthesis, as well as in vivo and in vitro research. An enzymatic assay can be used to determine the inhibitors' target specificity. Molecular docking studies could assist in identifying the inhibitors' orientation and binding interactions with the enzyme's active site. To evaluate drug likeness properties, the ADME property and oral toxicity were predicted. As a result, we expect this effort to provide some interesting molecules for the treatment of oxidative stress linked to a variety of pathological complications [20].

\section{Materials and Methods}

\subsection{Collection of Data Set}

Dataset of 27 ligands were taken in the present study [21]. All of the ligands' structures were drawn in ChemDraw Ultra 12.0 software [22] and saved in '.mol' format. The biological activity was reported in literature in terms of $\mathrm{IC}_{50}$ values (in $\left.\mu \mathrm{M}\right)$, which were converted to $\mathrm{pIC}_{50}$ for QSAR studies, as shown in Table 1. pIC50 is represented as the negative logarithmic value of IC50, so first the IC50 value was converted into molar concentration, 
then into $\mathrm{pIC} 50$ value. To generate the predicted $\mathrm{pIC}_{50}$ values for $3 \mathrm{D}-\mathrm{QSAR}$ analysis, the complete dataset was divided in 7:3 training and test sets. The complete process of the present study is depicted in Figure 1.

Table 1. Compounds taken in QSAR study with CA inhibitory activity ( $\mathrm{IC}_{50}$ and $\mathrm{pIC}_{50}$ value) [21].

\begin{tabular}{|c|c|c|c|c|}
\hline S. No. & Compounds & Structures & $\mathrm{IC}_{50}$ Value $(\mu \mathrm{M})$ & $\mathrm{pIC}_{50}$ Value \\
\hline 1 & $4 a$ & & 3 & 5.52 \\
\hline 2 & $4 b$ & & 8.03 & 5.52 \\
\hline 3 & $4 c$ & & 8.54 & 5.07 \\
\hline 4 & $4 d$ & & 26.16 & 5.07 \\
\hline 5 & $4 e$ & & 51.63 & 5.52 \\
\hline 6 & $4 \mathrm{f}$ & & 3.28 & 5.10 \\
\hline 7 & $4 \mathrm{~g}$ & & 18.74 & 5.07 \\
\hline 8 & $4 \mathrm{~h}$ & & 71.62 & 4.58 \\
\hline
\end{tabular}


Table 1. Cont.

\begin{tabular}{|c|c|c|c|c|}
\hline S. No. & Compounds & Structures & IC $_{50}$ Value $(\mu \mathrm{M})$ & $\mathrm{pIC}_{50}$ Value \\
\hline 9 & $4 i$ & & 2.55 & 4.29 \\
\hline 10 & $4 j$ & & 30.77 & 5.48 \\
\hline 11 & $4 k$ & & 222.82 & 4.73 \\
\hline 12 & 41 & & 7.11 & 4.14 \\
\hline 13 & $4 m$ & & 125 & 5.59 \\
\hline 14 & $4 n$ & & 9.09 & 4.51 \\
\hline 15 & 40 & & 4.32 & 3.65 \\
\hline 16 & $4 p$ & & 3.34 & 5.15 \\
\hline 17 & $4 q$ & & 4.31 & 3.90 \\
\hline
\end{tabular}


Table 1. Cont.

\begin{tabular}{|c|c|c|c|c|}
\hline S. No. & Compounds & Structures & IC $_{50}$ Value $(\mu \mathrm{M})$ & $\mathrm{pIC}_{50}$ Value \\
\hline 18 & $4 r$ & & 144.07 & 5.04 \\
\hline 19 & $4 s$ & & 42.25 & 5.36 \\
\hline 20 & $4 t$ & & 8.62 & 5.48 \\
\hline 21 & $4 u$ & & 4.34 & 5.37 \\
\hline 22 & $4 v$ & & 8.64 & 3.84 \\
\hline 23 & $4 w$ & & 73.25 & 4.37 \\
\hline 24 & $4 x$ & & 8.53 & 5.06 \\
\hline 25 & $5 a$ & & 7.89 & 5.36 \\
\hline
\end{tabular}


Table 1. Cont.

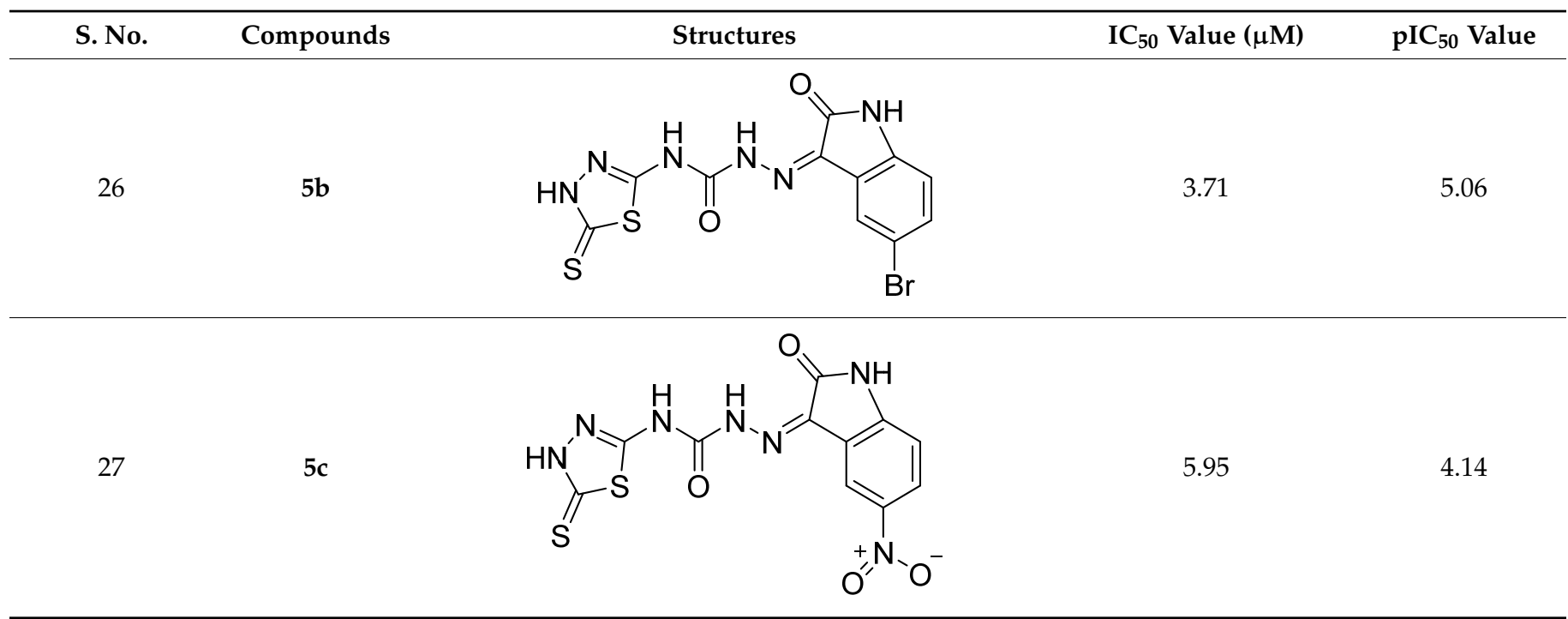

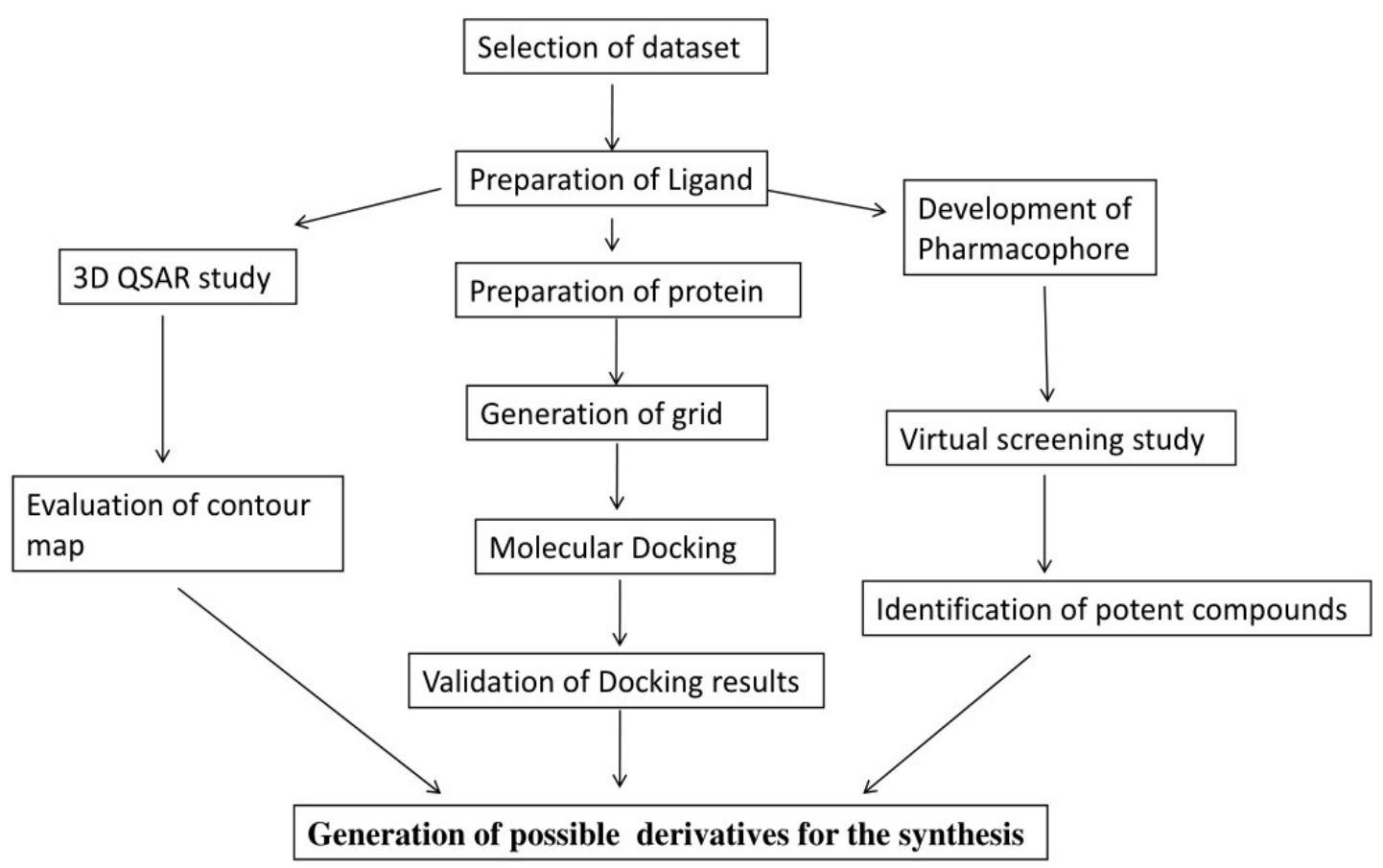

Figure 1. The schematic workflow of 3D QSAR, pharmacophore model, docking, and virtual screening performed in the present study.

\subsection{Preparation of Ligands}

The LigPrep [23] module was used to prepare the ligand via Maestro, which was specifically configured to provide input structures for the Glide and PHASE modules. Clean up wizard can process one ligand per second at a time, effectively converting large datasets from 2D to 3D structures, as well as essential steps in pharmacophore development and docking studies, utilising unique algorithms. To discover the optimal alignment and common features for 3D QSAR model generation, the molecules were aligned based on their most common core structure, as shown in Figure 2. 


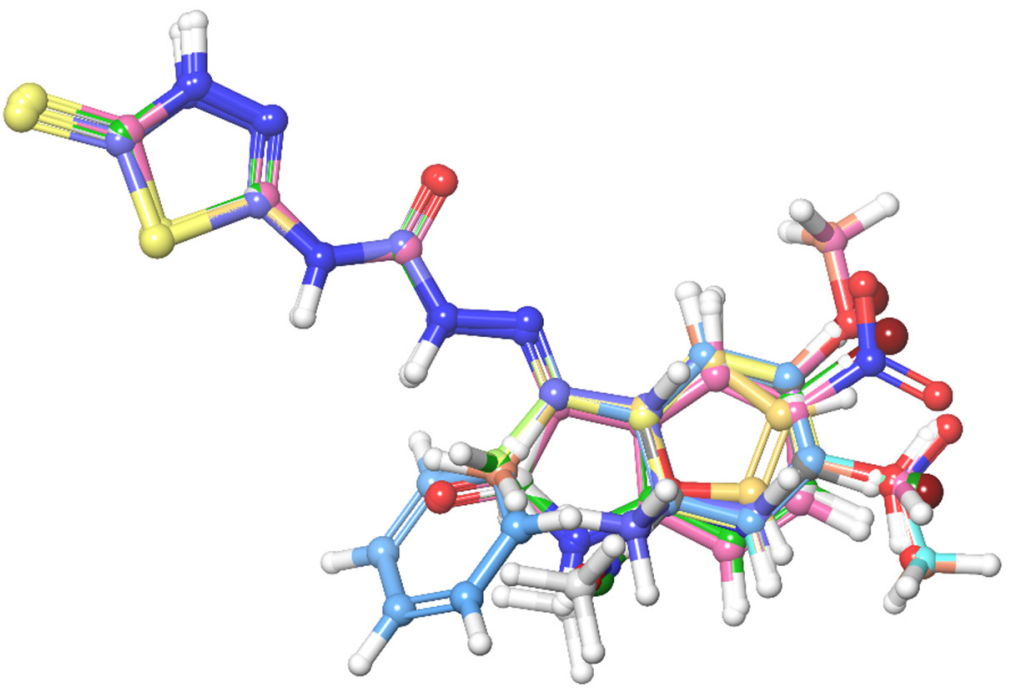

Figure 2. Molecular alignment of all the compounds taken in the QSAR study.

\subsection{Pharmacophore Mapping}

Pharmacophore mapping is the process of defining and placing unique pharmacophoric features as well as using alignment algorithms to overlay 3D conformations [24]. The PHASE module of the Schrodinger Maestro software was used to carry out the pharmacophore mapping study. There are various features present in developed pharmacophore hypothesis, such as hydrogen bond acceptors (A), hydrogen bond donor (D), aromatic ring $(\mathrm{R})$, and a hydrophobic $(\mathrm{H})$ group. The top ranked hypothesis consists of two unique pharmacophoric features based on structural requirements for biological activity: hydrogen bond donor (D) and ring aromaticity (R). As shown in Figure 3, each feature in this pharmacophoric map signifies a common particular portion associated with the selected compounds.

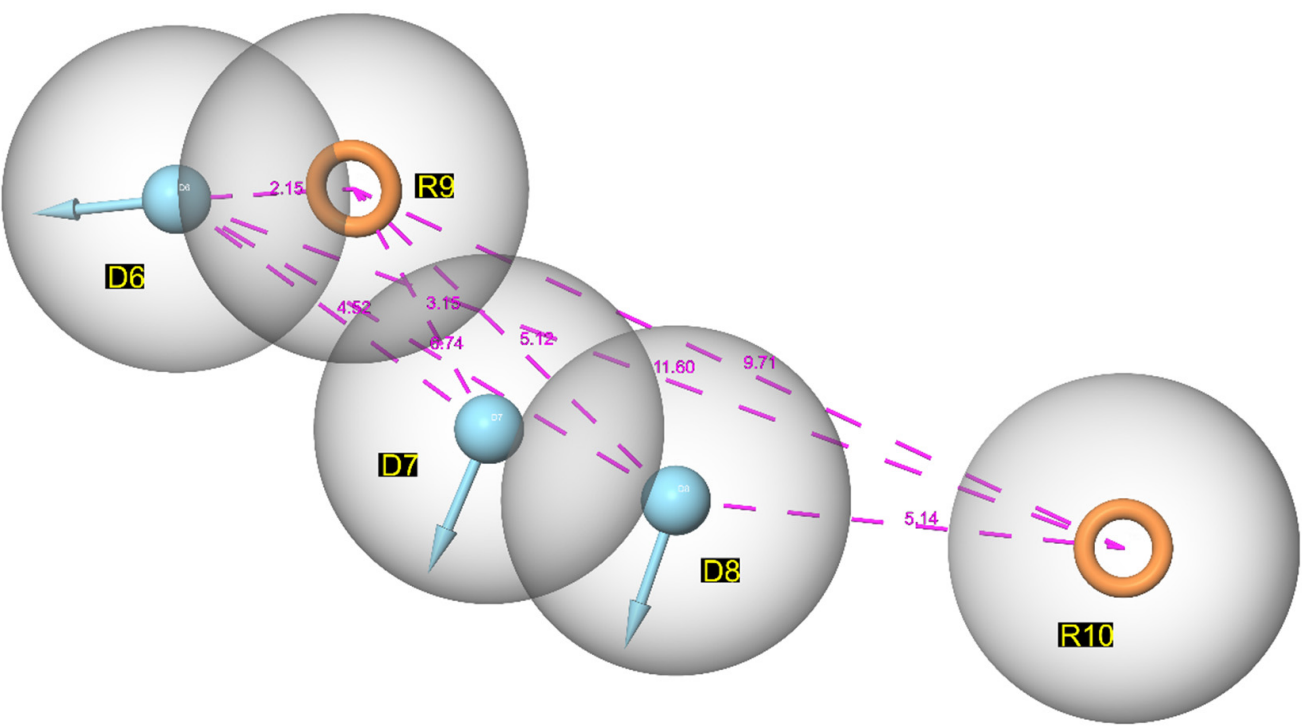

Figure 3. Common pharmacophoric features of generated module (DDDRR_1) with hydrogen bond donor (D) and ring aromaticity (R).

\subsection{Pharmacophore Hypothesis Generation}

Using the PHASE module's "Developing a pharmacophore model" [25,26], 20 hypotheses were created to explain how active molecules bind to receptors with a box size of 1 $\AA$ and a minimum inter site distance of $2 \AA$. A pharmacophore site is a characteristic feature of a conformation that has been mapped to a specific location. Common pharmacophoric 
features were identified from a set of variants-feature types that identify a putative pharmacophore. Using a scoring function as a survival score, the common pharmacophore hypotheses were investigated. The site, vector, volume, selectivity scores, and number of matches were calculated for each of the generated hypotheses. The PHASE module has six in-built pharmacophore features, including hydrogen bond acceptor (A), hydrogen bond donor $(\mathrm{D})$, hydrophobic group $(\mathrm{H})$, ring aromaticity $(\mathrm{R})$, positively ionizable $(\mathrm{P})$, and negatively ionizable $(\mathrm{N})$ groups (Supplementary File; Table S1).

\subsection{An Atom Based 3D-QSAR}

Schrodinger Maestro software was used to build an atom-based 3D-QSAR model using a collection of aligned ligands in order to predict activities for additional compounds [27]. The training set consisted of $70 \%$ of the dataset's compounds, while the test set consisted of $30 \%$. The compounds were clustered using a PLS factor of 4 . The Atom type fraction segment demonstrated the fraction owing to each atom type in the QSAR model for each number of PLS factors included in the model. Furthermore, actual activity versus predicted activity for compounds in the training as well as the test sets were plotted to form a scatter plot.

\subsection{Virtual Screening}

The virtual screening study was carried out using the ZINC data base, and the AAHRR 1 hypothesis was employed to screen ZINC compounds using the Lipinski rule of 5 (Supplementary File; Table S2) [28]. Using different filters, a total of 3568 molecules were screened. The GLIDE module of Schrodinger was used to screen these compounds using different docking methodologies. The Swiss Target Prediction tool was used to predict the target of these selected compounds. This tool predicts all the available targets of the molecule. Different targets were identified in the current investigation, with CA being the most important one for the particular molecule.

\subsection{Molecular Docking}

The docking studies were carried out using the Schrodinger Maestro software's Glide module [29]. The score function in the software was used to rank and group distinct possible adduct structures generated by molecular docking [30] (Supplementary File; Table S3). Based on the binding properties of the ligand and target, it predicts the three-dimensional structure of any complex. The method of predicting ligand conformation and orientation (or posing) within a specific binding site is referred to as docking. In Maestro wizard, the protein structure was pre-processed using the "protein preparation wizard". Hydrogen atoms and certain essential bonds were introduced to the missing site of the protein molecule by automatically generating states and refinement steps phases of the module. After the optimization process, receptor grid generation was processed and docking scores were analysed with different docked ligand conformations [31,32].

\subsection{ADME Properties Prediction}

The drug-like activity of the ligand molecule was predicted using the Schrodinger software's QikProp module [33]. It predicts both physicochemical descriptors and pharmacokinetic properties with the objective of increasing the success rate of compounds for further development. The ADME study suggests that drug-like properties of ligand molecules were validated using Lipinski's rule of five [34,35]. The rule states that the molecular weight $<500, \log \mathrm{P}_{\mathrm{o} / \mathrm{w}}<5$, hydrogen bond donor $\leq 5$, and hydrogen bond acceptor $\leq 10$. The compounds that meet these criteria are classified as drug-like compounds (Supplementary File; Tables S4 and S5). 


\section{Results and Discussion}

\subsection{Pharmacophore Mapping: Selection of the Best Pharmacophore Hypothesis}

All the selected compounds from the database were used to generate pharmacophoric hypothesis or the minimum features of the compound required to bind with receptor. Among 20 pharmacophore hypotheses, we screened best pharmacophoric hypotheses on the basis of various scores described in Table 2. Three hydrogen bond donors (D) and two ring aromaticity $(R)$ were identified as common pharmacophore features. DDDRR 1 and DDDRR 2 were found to be the best among the 20 hypotheses generated (Supplementary File; Table S1) by the PHASE module, based on a scoring function of each parameter value listed in Table 2 [36]. The "survival" scoring (S) function was responsible for determination of features from described models and to rank all hypotheses. The scoring algorithm involves selectivity, number of ligands matched, relative conformational energy, and activity. However, the models should also differentiate between inactive and active molecules. If an inactive molecule scores well, the hypothesis could be invalid as it does not discriminate between active and inactive molecules. For this reason, adjusted survival score S_I was calculated by subtracting the inactive score from the survival score.

Table 2. Pharmacophore hypothesis with ranking on the basis of survival scores.

\begin{tabular}{|c|c|c|c|c|c|c|c|c|c|}
\hline HypoID & Survival & Site & Vector & Volume & Select & Matches & Inactive & Adjusted & BEDROC \\
\hline DDDRR_1 & 5.4403 & 1 & 1 & 0.9301 & 1.9083 & 4 & 2.7979 & 2.6424 & 1 \\
\hline DDDRR_2 & 5.4403 & 1 & 1 & 0.9301 & 1.9083 & 4 & 2.7979 & 2.6424 & 1 \\
\hline DDDRR_3 & 5.4344 & 0.9999 & 1 & 0.9303 & 1.9021 & 4 & 2.8723 & 2.562 & 1 \\
\hline DDDRR_4 & 5.4336 & 1 & 1 & 0.9306 & 1.901 & 4 & 2.5881 & 2.8455 & 1 \\
\hline DDDRR_5 & 5.4233 & 1 & 1 & 0.9307 & 1.8906 & 4 & 2.5503 & 2.873 & 1 \\
\hline ADDRR_1 & 5.2355 & 1 & 1 & 0.93 & 1.7036 & 4 & 2.8242 & 2.4114 & 1 \\
\hline ADDRR_2 & 5.2177 & 1 & 1 & 0.9301 & 1.6857 & 4 & 2.898 & 2.3197 & 1 \\
\hline ADDRR_3 & 5.2028 & 1 & 1 & 0.8972 & 1.7036 & 4 & 2.7787 & 2.4241 & 1 \\
\hline ADDRR_4 & 5.1937 & 1 & 1 & 0.8979 & 1.6937 & 4 & 2.427 & 2.7667 & 1 \\
\hline ADDRR_5 & 5.1834 & 0.9999 & 1 & 0.9301 & 1.6513 & 4 & 2.8282 & 2.3553 & 1 \\
\hline DDRR_1 & 4.9585 & 1 & 1 & 0.9301 & 1.4264 & 4 & 2.8214 & 2.137 & 1 \\
\hline DDRR_2 & 4.9585 & 1 & 1 & 0.9301 & 1.4264 & 4 & 2.8214 & 2.137 & 1 \\
\hline DDRR_3 & 4.9527 & 1 & 1 & 0.9303 & 1.4204 & 4 & 2.9038 & 2.0489 & 1 \\
\hline DDRR_4 & 4.9489 & 1 & 1 & 0.9301 & 1.4168 & 4 & 2.7901 & 2.1588 & 1 \\
\hline DDRR_5 & 4.9488 & 1 & 1 & 0.9306 & 1.4162 & 4 & 2.6459 & 2.3029 & 1 \\
\hline DDRR_6 & 4.9475 & 1 & 1 & 0.9303 & 1.4153 & 4 & 2.8664 & 2.0812 & 1 \\
\hline DDRR_7 & 4.9451 & 0.9999 & 1 & 0.9303 & 1.4129 & 4 & 2.8672 & 2.0779 & 1 \\
\hline DDRR_8 & 4.9444 & 1 & 1 & 0.9307 & 1.4117 & 4 & 2.6223 & 2.3221 & 1 \\
\hline DDRR_9 & 4.9418 & 1 & 1 & 0.9306 & 1.4092 & 4 & 2.5853 & 2.3564 & 1 \\
\hline DDRR_10 & 4.9388 & 1 & 1 & 0.9307 & 1.4061 & 4 & 2.5917 & 2.3471 & 1 \\
\hline
\end{tabular}

\subsection{Selection of Atom Based QSAR Model}

The QSAR findings for the hypothesis are shown in the statistical table obtained for the training and test set molecules. Several statistical parameters were employed to examine the robustness of the QSAR model, including SD, $\mathrm{R}^{2}, \mathrm{~F}, \mathrm{P}, \mathrm{RMSE}, \mathrm{Q}^{2}$, and Pearson-R [37]. High $\mathrm{R}^{2}$ (higher than 0.6), $\mathrm{Q}^{2}$ (greater than 0.5), Pearson-R (greater than 0.5 ), and $F$ values characterize a good QSAR model. All the four models generated by the module are shown in Table 3. Based on the above information, the fourth model was chosen as the best QSAR model due to higher $\mathrm{Q}^{2}$ and $\mathrm{R}^{2}$ values as 0.7888 and 0.8757 , respectively.

To demonstrate the uniform distribution of training set molecules over the straight line passing through the origin $(0,0)$, a scattered plot was drawn between experimental activity and predicted activity of training and test set compounds (Figure 4). 
Table 3. Atom-based QSAR statistics with important values like $R^{\wedge} 2, Q^{\wedge} 2$ and $R^{\wedge} 2$ CV.

\begin{tabular}{|c|c|c|c|c|c|c|c|c|c|}
\hline \# Factors & SD & $\mathrm{R}^{\wedge} 2$ & $\mathrm{R}^{\wedge} 2 \mathrm{CV}$ & $\begin{array}{c}\mathbf{R}^{\wedge} 2 \\
\text { Scramble }\end{array}$ & $\mathbf{F}$ & $\mathbf{P}$ & RMSE & $Q^{\wedge} 2$ & Pearson-r \\
\hline 1 & 0.4137 & 0.474 & 0.043 & 0.3174 & 17.1 & 0.00056 & 0.6 & 0.2794 & 0.8494 \\
\hline 2 & 0.3767 & 0.5867 & 0.0764 & 0.4491 & 12.8 & 0.000352 & 0.51 & 0.4802 & 0.8547 \\
\hline 3 & 0.3599 & 0.8438 & 0.8096 & 0.549 & 10.2 & 0.000438 & 0.47 & 0.7448 & 0.8023 \\
\hline 4 & 0.3539 & 0.8757 & 0.8277 & 0.5943 & 58.3 & 0.000784 & 0.5 & 0.7888 & 0.7495 \\
\hline
\end{tabular}

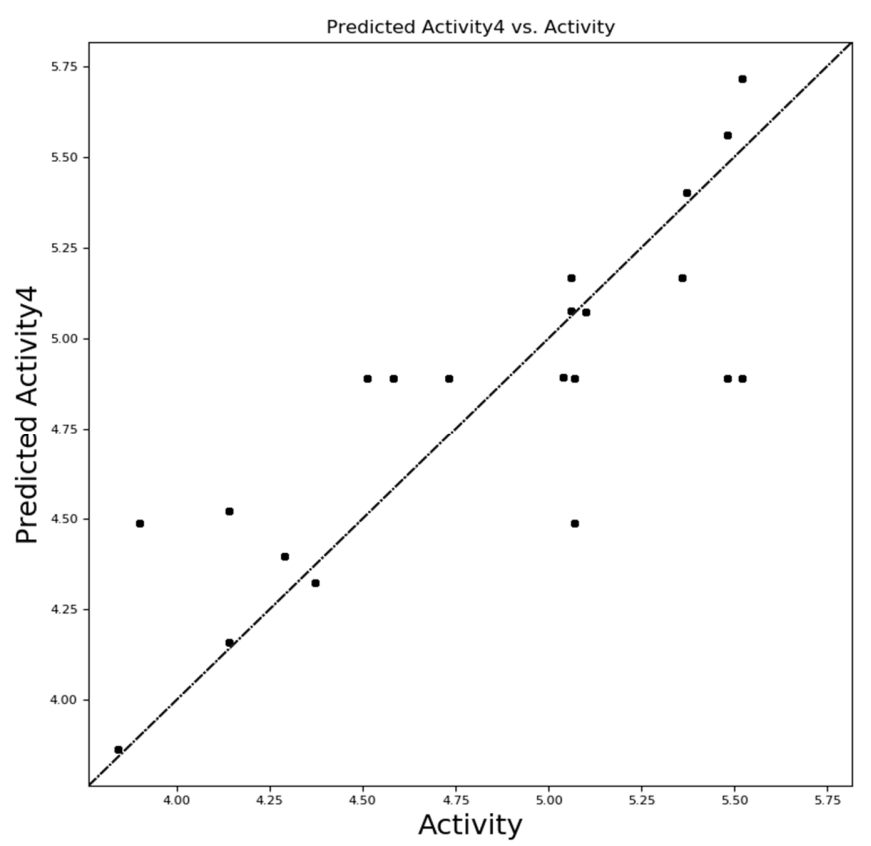

(A)

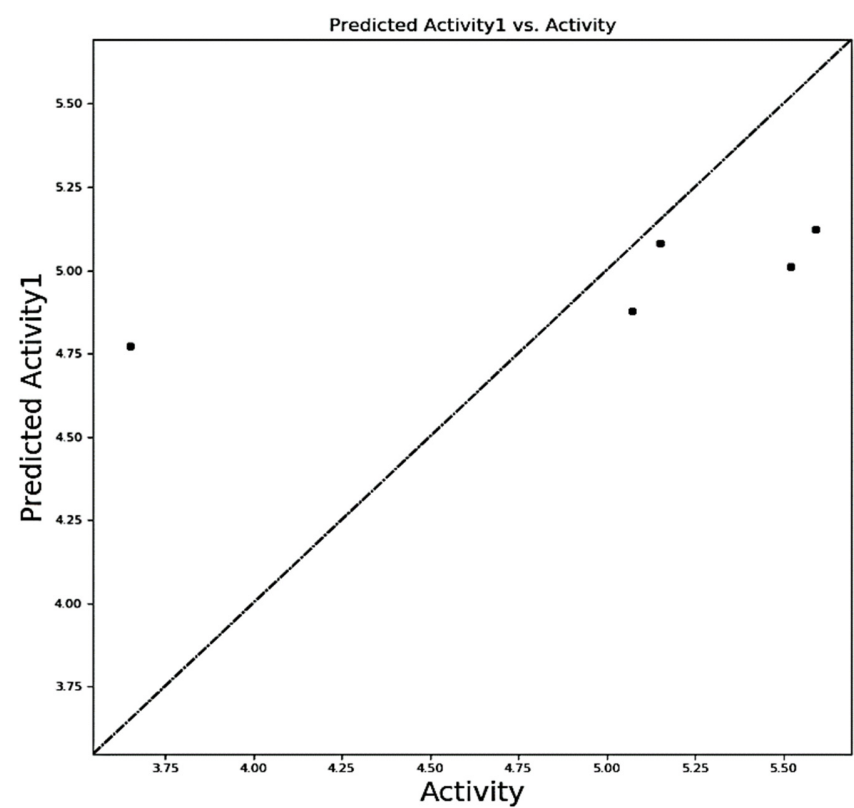

(B)

Figure 4. Scattered plot of experimental vs. predicted activity of training set (A) and test set (B) molecules, where the filled circles represent the training and test set compounds, respectively. 


\subsection{Evaluation of Contour Map}

The effects of different substituents on biological activity were determined by the study of contour maps, which are shown in Figure 5A-E. It also examines the variation of substituents and their biological activity. The contour map is represented by color coding, including different substituents on the core moiety. This color coding has a specific indication of the substituent and is useful in the expansion of novel compounds as CA inhibitors. The blue color showed enhanced activity, whereas the red color indicated decrease in activity. The electron-withdrawing group substitution on the phenyl ring showed a decrease in activity. Hydrogen bond donor group substitution on phenyl ring with para substitution showed an increase in activity. Hydrophobic substitution at the ortho position displayed good activity. Negative ionic group showed good activity at the para position and deceased activity in the meta position. The positive ionic group substitution in the phenyl ring showed decrease in activity.

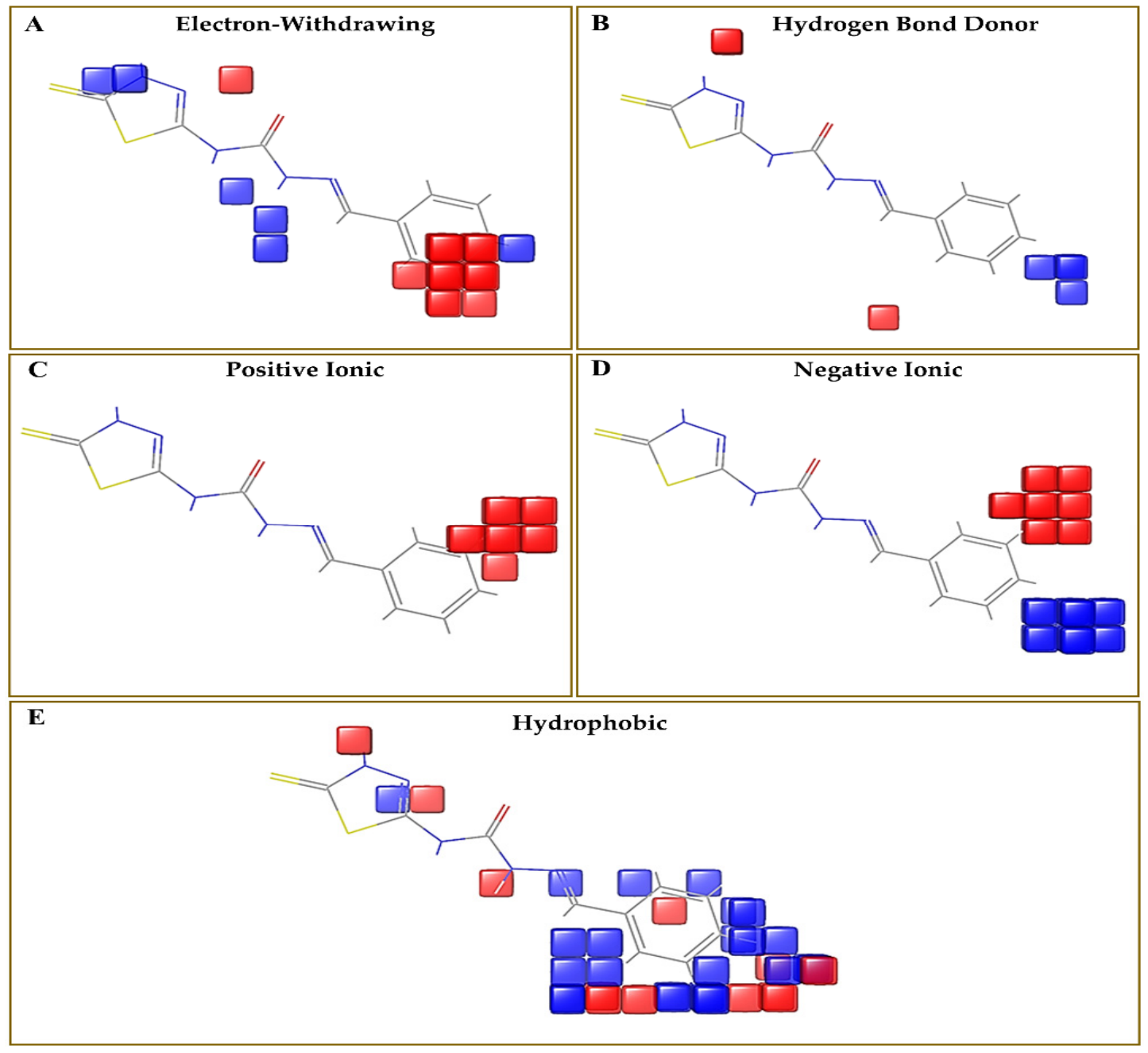

Figure 5. 3D-QSAR model (atom-based) consisting of (A) electron-withdrawing, (B) hydrogen bond donor, (C) positive ionic, (D) negative ionic, (E) hydrophobic groups, where the blue cubes showed positive coefficients and the red cubes showed negative coefficients.

\subsection{Molecular Docking Analysis}

The Glide module was utilized to conduct molecular docking between the potent derivatives and the target protein [38,39]. The potent analogues $4 \mathbf{m}, \mathbf{4 o}, \mathbf{4 s}, \mathbf{4} \mathbf{p}$, and $\mathbf{5 b}$ demonstrated the highest SP docking scores of $-5.217,-4.866,-4.729,-4.641$, and -4.635 respectively, when interacting with amino acids of the target protein. The docking scores of 
compounds in the dataset are shown in Table 4 . The purple arrows indicate hydrogen bond interactions, whereas the green arrows indicate $\pi$ - $\pi$-stacking interactions, as seen in Figure 6 for compound 4m, Figure 7A for compound 4o, and Figure 7B for compound 4s [40,41].

Table 4. Docking scores and MMGBSA-based rescores of compounds taken for QSAR and ZINC database.

\begin{tabular}{cccc}
\hline S. No. & Compound & Docking Score & $\begin{array}{c}\text { MMGBSA dG Bind (XPcomplex) } \\
\text { kcal/mol }\end{array}$ \\
\hline 1 & $\mathbf{4 m}$ & -5.217 & -72.8 \\
2 & $\mathbf{4 o}$ & -4.866 & -80.17 \\
3 & $\mathbf{4 s}$ & -4.729 & -74.67 \\
4 & $\mathbf{4 p}$ & -4.641 & -83.2 \\
5 & $\mathbf{5 b}$ & -4.635 & -76.8 \\
6 & ZINC77699643 & -6.178 & -69.8 \\
7 & ZINC89275054 & -5.743 & -84.17 \\
8 & ZINC77671412 & -5.561 & -67.67 \\
9 & ZINC70762033 & -5.535 & -82.2 \\
\hline
\end{tabular}

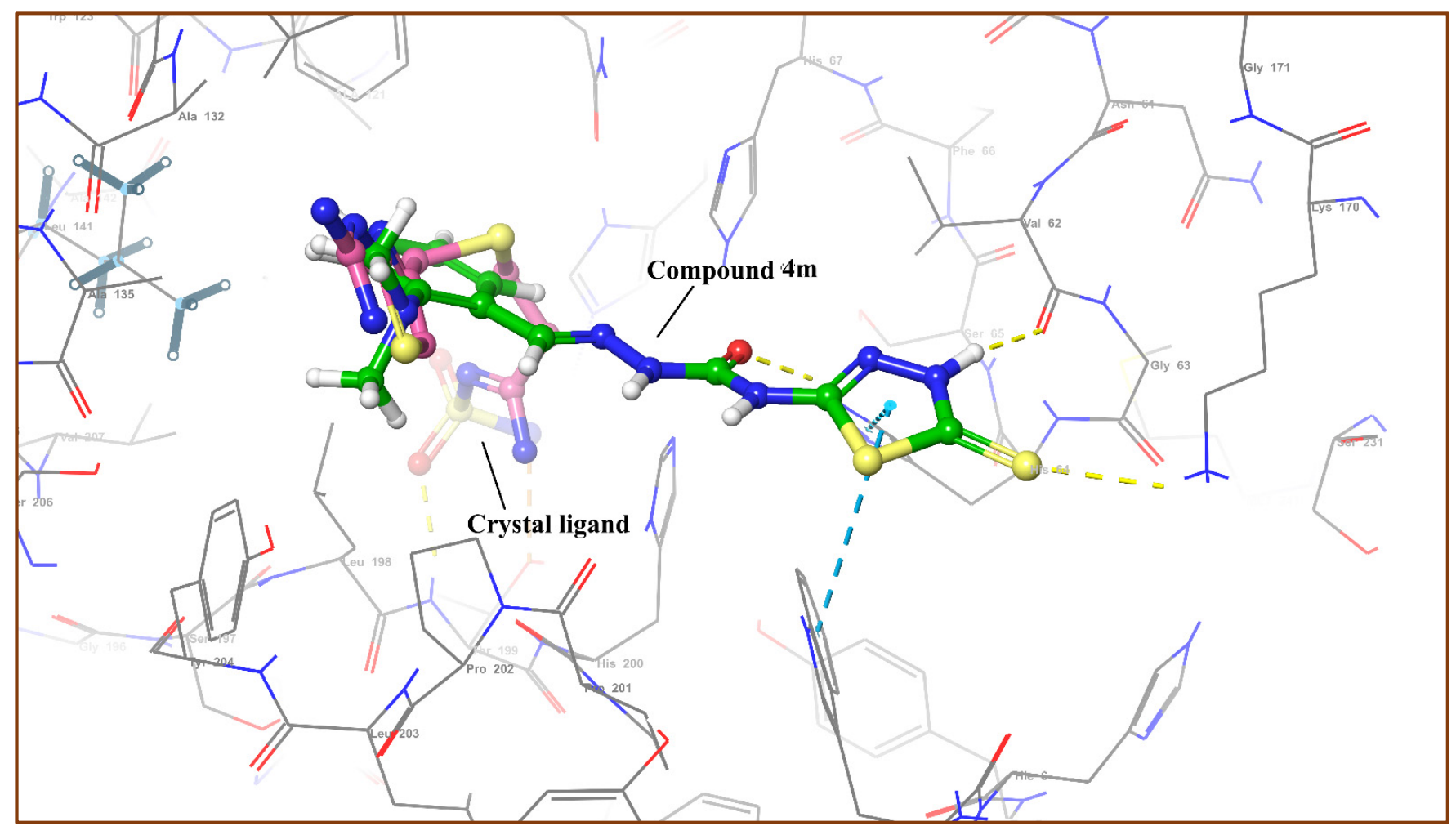

Figure 6. 3D interactions of compound $4 \mathrm{~m}$ and crystal ligand with different amino acids His64, Val62 at the binding site of receptor $6 \mathrm{~g} 3 \mathrm{v}$.

\subsection{Virtual Screening}

The molecular docking methodologies such as HTVS, SP, and XP were applied to screen the compounds from the ZINC database. Each step screened 20\% of the highestscoring compounds with high docking scores. The top compounds, ZINC77699643, ZINC89275054, ZINC77671412, and ZINC70762033, were selected with xp docking scores of $-6.178,-5.743,-5.561$, and -5.535 , respectively, out of 333 compounds screened using the SP docking approach. These compounds were chosen as the final ZINC compounds for further investigation and assessed using MMGBSA to calculate binding interaction energy. The compound ZINC77699643 interacted with Pro201 and Thr199 amino acid residues, as shown in Figure 8A. These interactions demonstrated the significance of CA activity. 
The compound ZINC89275054 showed binding interactions with amino acid residues His94, Gln92, and Thr199 in the same cavity, as shown by a crystal ligand (Figure 8B). The compound ZINC77671412 demonstrated binding interactions with several amino acids at the receptor's binding region, including His200 and Gln92, which are critical for activity (Figure 9A). The compound ZINC70762033 showed binding interaction with different amino acids such as His200, His94, Gln92, and Thr199 at the binding site of receptor (Figure 9B).
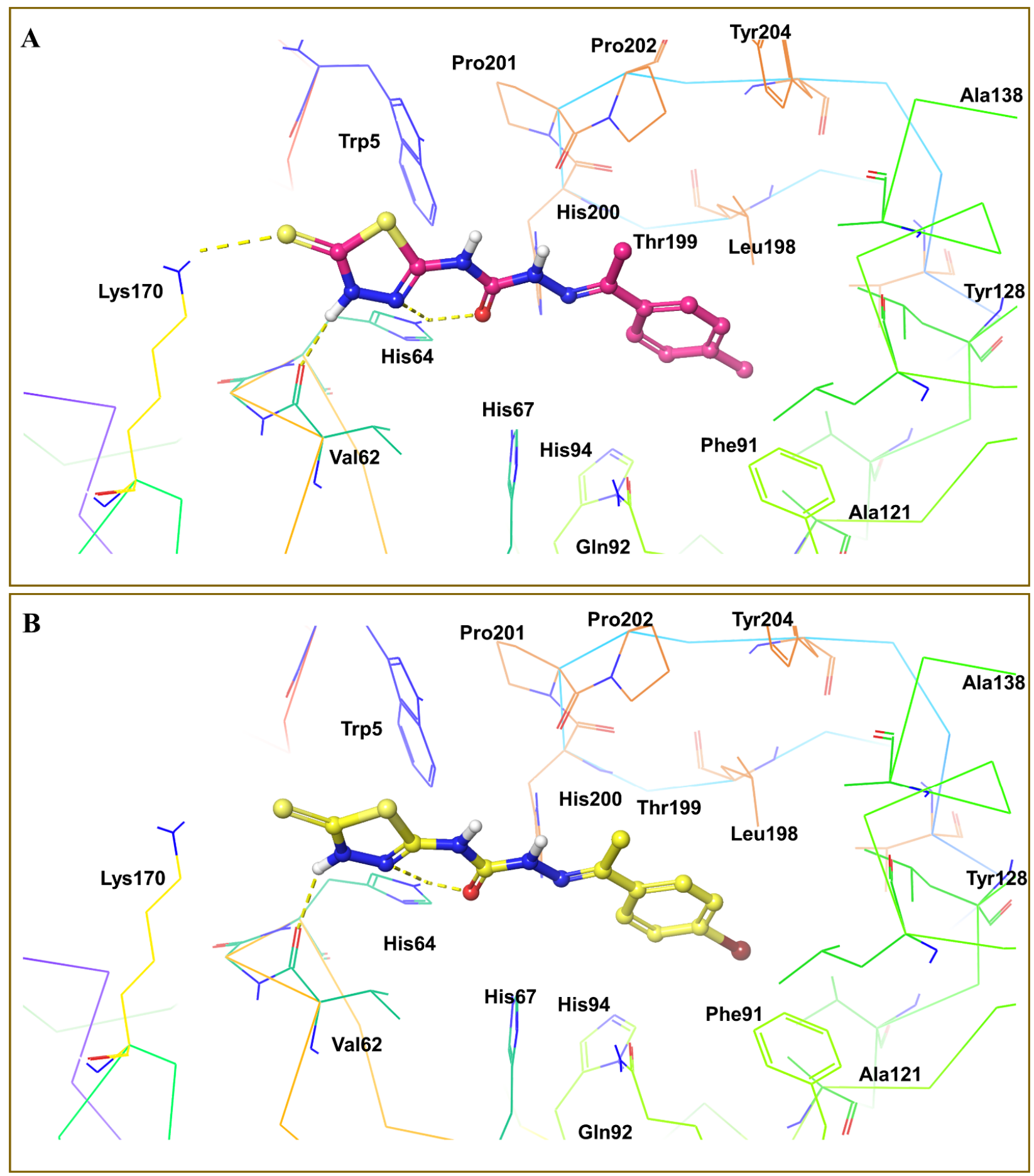

Figure 7. (A) 3D interactions of compound 4o with different amino acids His64, Val62, Lys170 at the binding site of receptor $6 \mathrm{~g} 3 \mathrm{v}$. (B) 3D interactions of compound 4s with different amino acids His64, and Val62 at the binding site of receptor $6 \mathrm{~g} 3 \mathrm{v}$. 

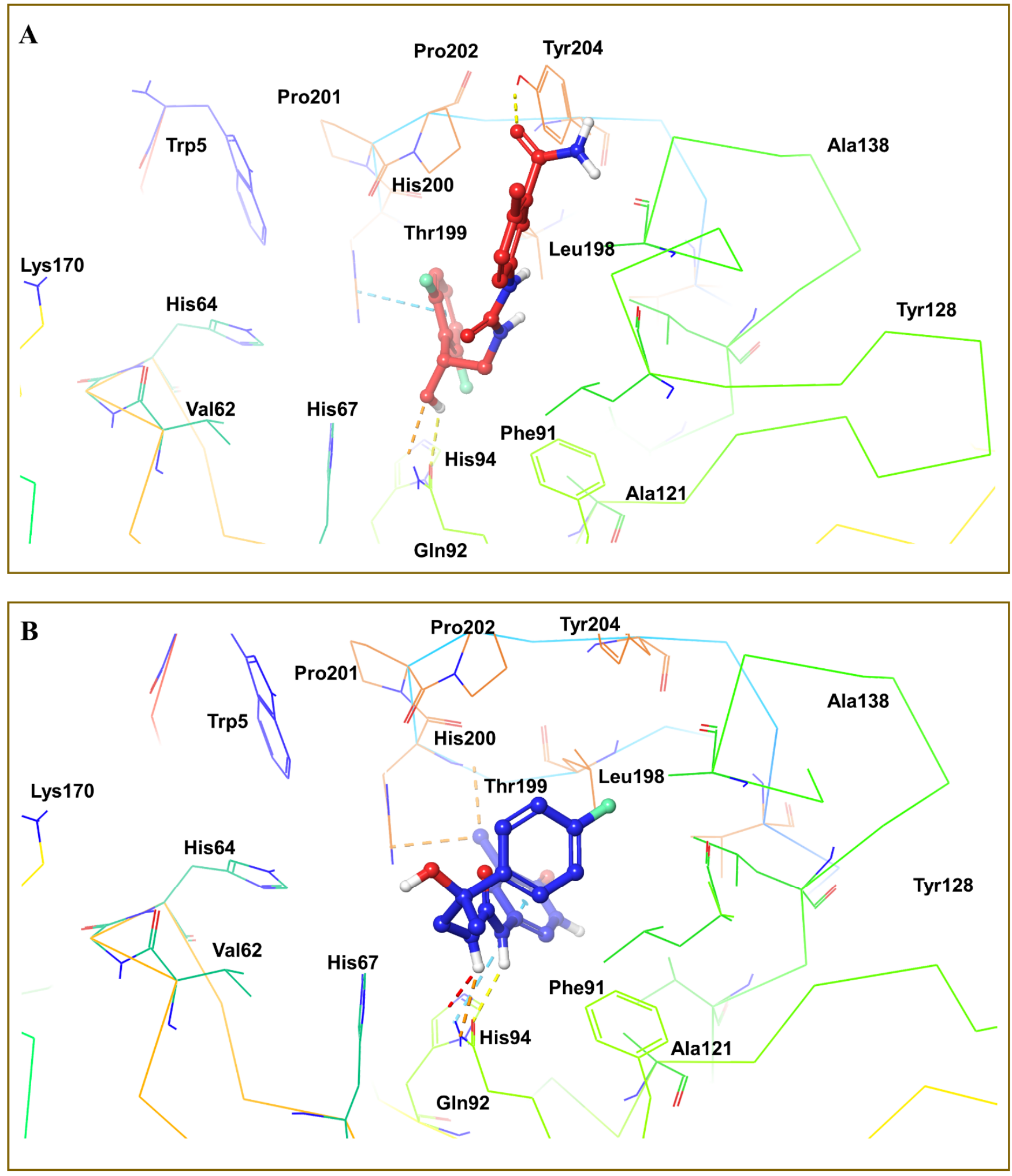

Figure 8. (A) 3D interactions of compound ZINC77699643 with different amino acids-Pro201 and Thr199-at the binding site of receptor 6g3v. (B) 3D interactions of compound ZINC89275054 with different amino acids_-His94, Gln92, and Thr199_-at the binding site of receptor $6 \mathrm{~g} 3 \mathrm{v}$. 

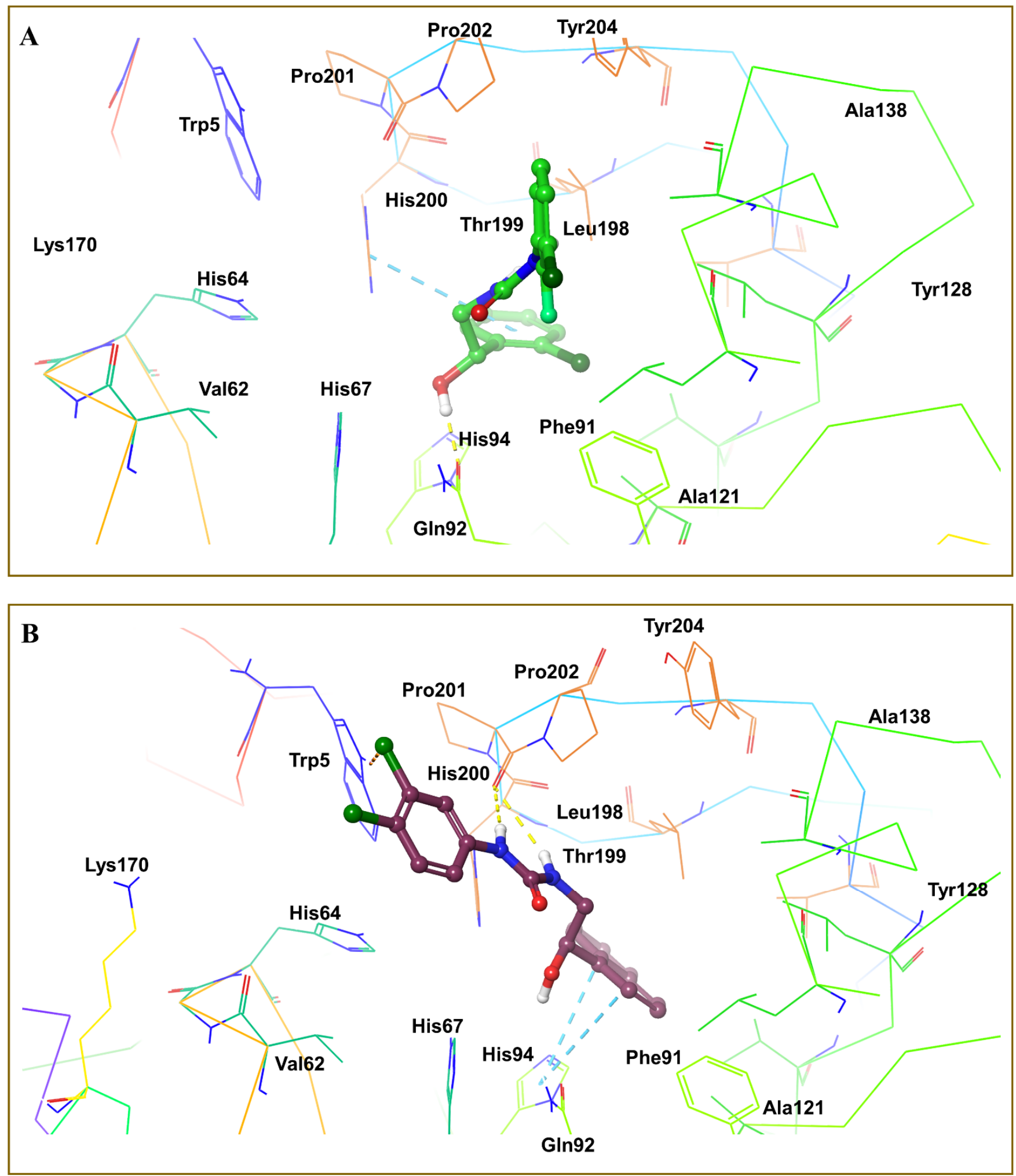

Figure 9. (A) 3D interactions of compound ZINC77671412 with different amino acids-His200 and Gln92 - at the binding site of receptor 6g3v. (B) 3D interactions of compound ZINC70762033 with different amino acids-His200, His94, Gln92, and Thr199-at the binding site of receptor 6g3v.

\subsection{ADME Properties Prediction}

The ADME properties of all the compounds from the dataset were predicted through the QikProp module of Schrodinger; the results are given in Table 5. The software predicted 
the compounds' physicochemical properties, lipophilicity, drug-like behaviour, water solubility, permeability through BBB, pharmacokinetics, and synthetic accessibility [42,43]. All compounds have a molecular weight ranging from 259.34 to 399.24 , number of hydrogen bond acceptors ranging from 4.5 to 7.0 , and number of hydrogen bond donors ranging from 3 to 4 . All the predicted ADME properties satisfied the Lipinski's rule of five for all the compounds.

Table 5. Result of ADME properties of compounds taken for QSAR analysis.

\begin{tabular}{|c|c|c|c|c|c|c|c|c|}
\hline Compound & CNS & MW $(<500)$ & Dipole & HBD $(<5)$ & HBA $(<10)$ & $\begin{array}{c}\text { QPlogPo/w } \\
(\leq 5)\end{array}$ & $\begin{array}{c}\text { Rule of } \\
\text { Five }(\leq 1)\end{array}$ & $\begin{array}{c}\text { Rule of } \\
\text { Three }\end{array}$ \\
\hline $4 a$ & -1 & 279.334 & 8.68 & 3 & 5 & 1.331 & 0 & 0 \\
\hline $4 b$ & -2 & 295.333 & 7.564 & 4 & 5.75 & 0.601 & 0 & 0 \\
\hline $4 c$ & -2 & 309.36 & 7.389 & 3 & 5.75 & 1.447 & 0 & 0 \\
\hline $4 d$ & -2 & 309.36 & 9.912 & 3 & 5.75 & 1.446 & 0 & 0 \\
\hline $4 e$ & -2 & 325.36 & 8.614 & 4 & 6.5 & 0.754 & 0 & 0 \\
\hline $4 f$ & -1 & 313.779 & 7.85 & 3 & 5 & 1.744 & 0 & 0 \\
\hline $4 g$ & -1 & 313.779 & 7.342 & 3 & 5 & 1.805 & 0 & 0 \\
\hline $4 h$ & -1 & 358.23 & 9.087 & 3 & 5 & 1.878 & 0 & 0 \\
\hline $4 i$ & -1 & 358.23 & 7.462 & 3 & 5 & 1.878 & 0 & 0 \\
\hline $4 j$ & -1 & 297.325 & 7.295 & 3 & 5 & 1.558 & 0 & 0 \\
\hline $4 \mathrm{k}$ & -2 & 324.332 & 7.092 & 3 & 6 & 0.713 & 0 & 0 \\
\hline 41 & -2 & 324.332 & 6.573 & 3 & 6 & 0.662 & 0 & 0 \\
\hline $4 m$ & -2 & 322.402 & 8.929 & 3 & 6 & 1.811 & 0 & 0 \\
\hline $4 n$ & -1 & 293.361 & 9.244 & 3 & 4.5 & 1.753 & 0 & 0 \\
\hline 40 & -1 & 307.388 & 9.628 & 3 & 4.5 & 2.037 & 0 & 0 \\
\hline $4 p$ & -2 & 309.36 & 10.476 & 4 & 5.25 & 1.009 & 0 & 0 \\
\hline $4 q$ & $-\overline{1}$ & 323.387 & 10.496 & 3 & 5.25 & 1.907 & 0 & 0 \\
\hline $4 r$ & -1 & 327.806 & 7.953 & 3 & 4.5 & 2.231 & 0 & 0 \\
\hline $4 \mathrm{~s}$ & -1 & 372.257 & 8.067 & 3 & 4.5 & 2.305 & 0 & 0 \\
\hline $4 t$ & -2 & 338.358 & 7.204 & 3 & 5.5 & 1.079 & 0 & 0 \\
\hline $4 u$ & -1 & 355.432 & 9.928 & 3 & 4.5 & 2.994 & 0 & 0 \\
\hline $4 v$ & -2 & 280.322 & 6.159 & 3 & 6.5 & 0.678 & 0 & 0 \\
\hline $4 w$ & -1 & 269.296 & 8.4 & 3 & 5.5 & 0.734 & 0 & 0 \\
\hline $4 x$ & -2 & 259.344 & 9.12 & 3 & 5 & 0.822 & 0 & 0 \\
\hline $5 a$ & -2 & 320.343 & 10.649 & 3 & 6 & 0.683 & 0 & 0 \\
\hline $5 b$ & -2 & 399.239 & 10.195 & 3 & 6 & 1.227 & 0 & 0 \\
\hline $5 c$ & -2 & 365.341 & 10.978 & 3 & 7 & 0.016 & 0 & 0 \\
\hline
\end{tabular}

\subsection{MMGBSA-Based Rescoring}

The MMGBSA-based rescoring method was used for calculation of binding free energy for ligands and ZINC hit compounds ZINC77699643, ZINC89275054, ZINC77671412, and ZINC70762033 (complex with PDB ID: 6g3v), which provided very high binding free energy, as $\mathrm{dG}$ bind $-69.8,-84.17,-67.67,-82.2 \mathrm{kcal} / \mathrm{mol}$, respectively (Table 4 ).

\section{Optimization of Novel Ligands}

Optimization of ligands revealed that the substitution of the hydrogen bond donor group on the phenyl ring at the para position, hydrophobic group at the ortho position, and negative ionic group at the para position showed increase in activity. However, substitution of the negative ionic group at the meta position of the phenyl ring, and positive ionic group substitution on the phenyl ring displayed a decrease in activity (Figure 10). 
Negative ionic group at para position increases activity, whereas decreases at meta position

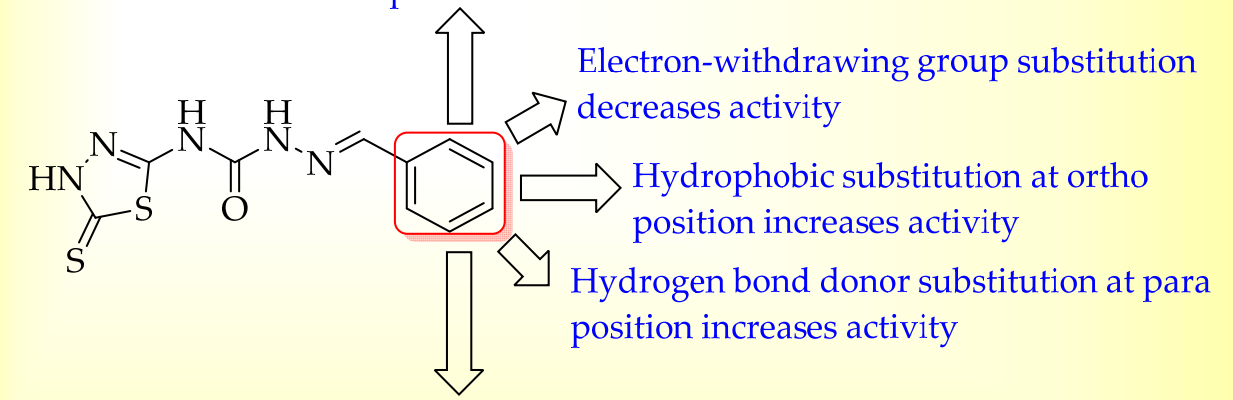

Positive ionic group substitution decreases activity

Figure 10. Optimization of ligands for the development of novel thiadiazole derivatives.

\section{Conclusions}

The computational design of 1,3,4-thiadiazole derivatives as potential CA inhibitors was discussed in this study. The PHASE module (Schrodinger) was used to investigate the pharmacophore model on 27 compounds taken from a dataset. The compound $4 \mathbf{m}$ [(2-(2(dimethylamino)benzylidene)- $N$-(5-thioxo-4,5-dihydro-1,3,4-thiadiazol-2-yl)hydrazine-1carboxamide)] showed the best SP docking score of -5.217 . The predicted ADME properties revealed that all the compounds followed the Lipinski's rule of five, indicating their druglike potential. Optimization of ligands can help to generate novel thiadiazole derivatives by using the response of a contour map. The generated contour map showed the substitution of the electron-withdrawing group on the phenyl ring responsible for decrease in CA activity. However, the hydrophobic group substitution at the ortho position, and hydrogen bond donor substitution at the para position of the phenyl ring showed an increase in CA activity. These results may aid in the development of novel thiadiazole compounds as CA inhibitors, which might have a major influence on the treatment of oxidative stress induced by low oxygen pressure at high altitudes.

Supplementary Materials: The following supporting information can be downloaded at:https:// www.mdpi.com/article/10.3390/cimb44030068/s1, Table S1: The pharmacophore study carried out for the dataset; Table S2: The HTVS docking study carried out for the ZINC compounds; Table S3: The docking study carried out for the dataset; Table S4: The QIKPROP study carried out the ZINC database; Table S5: The ADME study carried out for the database.

Author Contributions: Conceptualization, A.A. (Amena Ali) and A.A. (Abuzer Ali); methodology, A.A. (Amena Ali) and F.A.; investigation, M.J.A. and F.A.; resources, A.A. (Amena Ali) and A.A. (Abuzer Ali); data curation, A.A. (Amena Ali) and F.A.; writing-original draft preparation, M.J.A.; A.A. (Amena Ali) and A.A. (Abuzer Ali); writing-review and editing, M.H.W. and M.A.R.; supervision, A.A. (Amena Ali) and A.A. (Abuzer Ali); project administration, A.A. (Amena Ali); funding acquisition, A.A. (Amena Ali). All authors have read and agreed to the published version of the manuscript.

Funding: This work is supported by grants from the High-Altitude Research Center, Taif University, Taif, Saudi Arabia (Grant number: 1-442-45).

Institutional Review Board Statement: Not applicable.

Informed Consent Statement: Not applicable.

Data Availability Statement: The authors confirm that the data supporting the study's findings are included in the article and its Supplementary Information. 
Acknowledgments: The authors would like to extend their sincere thanks to High Altitude Research Center, Taif University, for its funding of this research through the Research Group, Project number: $1-442-45$.

Conflicts of Interest: The authors declare no conflict of interest.

\section{References}

1. Nita, M.; Grzybowski, A. The Role of the Reactive Oxygen Species and Oxidative Stress in the Pathomechanism of the Age-Related Ocular Diseases and Other Pathologies of the Anterior and Posterior Eye Segments in Adults. Oxid. Med. Cell Longev. 2016, 2016, 3164734. [CrossRef]

2. Therond, P. Dommagescréés aux biomolécules (lipides, protéines, ADN) par le stress oxydant [Oxidative stress and damages to biomolecules (lipids, proteins, DNA)]. Ann. Pharm. Fr. 2006, 64, 383-389. [CrossRef]

3. Pacifici, R.E.; Davies, K.J. Protein, lipid and DNA repair systems in oxidative stress: The free-radical theory of aging revisited. Gerontology 1991, 37, 166-180. [CrossRef] [PubMed]

4. Aslam, S.; Gupta, V. Carbonic Anhydrase Inhibitors. In StatPearls [Internet]; StatPearls Publishing: Treasure Island, FL, USA, 2021. Available online: https:/ / www.ncbi.nlm.nih.gov/books/NBK557736/ (accessed on 10 January 2022).

5. Supuran, C.T. An update on drug interaction considerations in the therapeutic use of carbonic anhydrase inhibitors. Exp. Opin. Drug Metabol. Tox. 2020, 16, 297-307. [CrossRef] [PubMed]

6. Angeli, A.; Supuran, C.T. Treatment of sleep apnea with a combination of a carbonic anhydrase inhibitor and an aldosterone antagonist: A patent evaluation of CA2958110 and IN6616DEN2012. Exp. Opin. Ther. Pat. 2018, 28, 723-727. [CrossRef] [PubMed]

7. Di Meo, S.; Reed, T.T.; Venditti, P.; Victor, V.M. Role of ROS and RNS Sources in Physiological and Pathological Conditions. Oxid. Med. Cell Longev. 2016, 2016, 1245049. [CrossRef] [PubMed]

8. Dixon, S.L.; Smondyrev, A.M.; Knoll, E.H.; Rao, S.N.; Shaw, D.E.; Friesner, R.A. PHASE: A new engine for pharmacophore perception, 3D QSAR model development, and 3D database screening: 1. Methodology and preliminary results. J. Comput. Aided Mol. Des. 2006, 20, 647-671. [CrossRef]

9. Pandey, R.K.; Kumbhar, B.V.; Sundar, S.; Kunwar, A.; Prajapati, V.K. Structure-based virtual screening, molecular docking, ADMET and molecular simulations to develop benzoxaborole analogs as potential inhibitor against Leishmania donovani trypanothione reductase. J. Recept. Signal Transduct. 2017, 37, 60-70. [CrossRef]

10. Bakonyi, T.; Radak, Z. High Altitude and Free Radicals. J. Sports Sci. Med. 2004, 3, 64-69.

11. Supuran, C.T. Carbonic anhydrase inhibitors as emerging drugs for the treatment of obesity. Expert. Opin. Emerg. Drugs 2012, 17, 11-15. [CrossRef]

12. Forwand, S.A.; Landowne, M.; Follansbee, J.N.; Hansen, J.E. Effect of acetazolamide on acute mountain sickness. N. Engl. J. Med. 1968, 279, 839-845. [CrossRef] [PubMed]

13. Secil, D.; Tugba, K.U.; Clemente, C.; Claudiu, T.S.; Ozen, O.G. Is carbonic anhydrase inhibition useful as a complementary therapy of Covid-19 infection? , J. Enzyme Inhib. Med. Chem. 2021, 36, 1230-1235. [CrossRef]

14. Sechi, M.; Innocenti, A.; Pala, N.; Rogolina, D.; Carcelli, M.; Scozzafava, A.; Supuran, C.T. Inhibition of $\alpha$-class cytosolic human carbonic anhydrases I, II, IX and XII, and $\beta$-class fungal enzymes by carboxylic acids and their derivatives: New isoform-I selective nanomolar inhibitors. Bioorg. Med. Chem. Lett. 2012, 22, 5801-5806. [CrossRef] [PubMed]

15. Pastorekova, S.; Zatovicova, M.; Pastorek, J. Cancer-associated carbonic anhydrases and their inhibition. Curr. Pharm. Des. 2008, 14, 685-698. [CrossRef]

16. Carradori, S.; Mollica, A.; De Monte, C.; Ganese, A.; Supuran, C.T. Nitric oxide donors and selective carbonic anhydrase inhibitors: A dual pharmacological approach for the treatment of glaucoma, cancer and osteoporosis. Molecules 2015, 20, 5667-5679. [CrossRef]

17. Jain, A.K.; Sharma, S.; Vaidya, A.; Ravichandran, V.; Agarwal, R.K. 1,3,4-Thiadiazole and its derivatives: A review on recent progress in biological activities. Chem. Biol. Drug Des. 2015, 81, 557-576. [CrossRef]

18. Patel, C.N.; Georrge, J.J.; Modi, K.M.; Narechania, M.B.; Patel, D.P.; Gonzalez, F.J.; Pandya, H.A. Pharmacophore-based virtual screening of catechol-o-methyltransferase (COMT) inhibitors to combat Alzheimer's disease. J. Biomol. Struct. Dyn. 2018, 36, 3938-3957. [CrossRef]

19. Grüneberg, S.; Stubbs, M.T.; Klebe, G. Successful virtual screening for novel inhibitors of human carbonic anhydrase: Strategy and experimental confirmation. J. Med. Chem. 2002, 45, 3588-3602. [CrossRef]

20. Ferdinando, G.; Brownlee, M. Oxidative stress and diabetic complications. Circ. Res. 2010, 107, 1058-1070.

21. Abdel-Hamid, M.K.; Abdel-Hafiz, A.A.; El-koussi, N.A.; Mahfouz, N.M.; Innoceti, A.; Supuran, C.T. Design, synthesis, and docking studies of new 1,3,4-thiadiazole-2-thione derivatives with carbonic anhydrase inhibitory activity. Bioorg. Med. Chem. 2007, 15, 6975-6984. [CrossRef]

22. Mills, N. ChemDraw Ultra 10.0. J. Am. Chem. Soc. 2006, 128, 13649-13650. [CrossRef]

23. LigPrep; Schrödinger, LLC: New York, NY, USA, 2021.

24. Seidel, T.; Ibis, G.; Bendix, F.; Wolber, G. Strategies for 3D pharmacophore-based virtual screening. Drug Discov. Today Technol. 2010, 7, e221-e228. [CrossRef] [PubMed]

25. Phase; Schrödinger, LLC: New York, NY, USA, 2021. 
26. Zhang, W.; Koehler, K.F.; Zhang, P.; Cook, J.M. Development of a comprehensive pharmacophore model for the benzodiazepine receptor. Drug Des. Discov. 1995, 12, 193-248. [PubMed]

27. Sippl, W. Receptor-based 3D QSAR analysis of estrogen receptor ligands-merging the accuracy of receptor-based alignments with the computational efficiency of ligand-based methods. J. Comput. Aided Mol. Des. 2000, 14, 559-572. [CrossRef] [PubMed]

28. Irwin, J.J.; Shoichet, B.K. ZINC-A free database of commercially available compounds for virtual screening. J. Chem. Inf. Model 2005, 45, 177-182. [CrossRef]

29. Glide; Schrödinger, LLC: New York, NY, USA, 2021.

30. Friesner, R.A.; Banks, J.L.; Murphy, R.B.; Halgren, T.A.; Klicic, J.J.; Mainz, D.T.; Repasky, M.P.; Knoll, E.H.; Shelley, M.; Perry, J.K.; et al. Glide: A New Approach for Rapid, Accurate Docking and Scoring. 1. Method Assessment Docking Accuracy. J. Med. Chem. 2004, 47, 1739-1749. [CrossRef]

31. Asati, V.; Bharti, S.K.; Das, D.; Kashaw, V.; Kashaw, S.K. Discovery of novel ALK2 inhibitors of pyrazolo-pyrimidines: A computational study. J. Biomol. Struct. Dyn. 2021, 1-15. [CrossRef]

32. Bhole, R.P.; Bonde, C.G.; Bonde, S.C.; Chikhale, R.V.; Wavhale, R.D. Pharmacophore model and atom-based 3D quantitative structure activity relationship (QSAR) of human immunodeficiency virus-1 (HIV-1) capsid assembly inhibitors. J. Biomol. Struc. Dyn. 2021, 39, 718-727. [CrossRef]

33. QikProp; Schrödinger, LLC: New York, NY, USA, 2021.

34. Di, L.; Kerns, E. Drug-like Properties: Concepts, Structure Design and Methods from ADME to Toxicity Optimization; Academic Press: Cambridge, MA, USA, 2015.

35. Nogara, P.A.; De-Aquino Saraiva, R.; Bueno, D.C.; Lissner, L.J.; Corta, C.L.D.; Braga, M.M.; Rosemberg, D.B.; Rocha, J.B.T. Virtual screening of acetylcholinesterase inhibitors using the Lipinski's rule of five and ZINC databank. BioMed Res. Int. 2015, 2015, 870389. [CrossRef]

36. Pal, S.; Kumar, V.; Kundu, B.; Bhattacharya, D.; Preethy, N.; Reddy, M.P.; Talukdar, A. Ligand-based pharmacophore modeling, virtual screening and molecular docking studies for discovery of potential topoisomerase I inhibitors. Comput. Struc. Biotech. J. 2019, 17, 291-310. [CrossRef]

37. Shah, U.A.; Deokar, H.S.; Kadam, S.S.; Kulkarni, V.M. Pharmacophore generation and atom-based 3D-QSAR of novel 2-(4methylsulfonylphenyl) pyrimidines as COX-2 inhibitors. Mol. Divers. 2010, 14, 559-568. [CrossRef] [PubMed]

38. Lalit, M.; Gangwal, R.P.; Dhoke, G.V.; Damre, M.V.; Khandelwal, K.; Sangamwar, A.T. A combined pharmacophore modeling, 3D-QSAR and molecular docking study of substituted bicyclo-[3.3.0] oct-2-enes as liver receptor homolog-1 (LRH-1) agonists. J. Mol. Stru. 2013, 1049, 315-325. [CrossRef]

39. Ghalla, H.; Issaoui, N.; Bardak, F.; Atac, A. Intermolecular interactions and molecular docking investigations on 4methoxybenzaldehyde. Comput. Mat. Sci. 2018, 149, 291-300. [CrossRef]

40. Warren, G.L.; Andrews, W.; Capelli, A.M.; Clarke, B.; LaLonde, J.; Lamber, M.H.; Lindvall, M.; Nevins, N.; Semus, S.F.; Senger, S.; et al. A critical assessment of docking programs and scoring functions. J. Med. Chem. 2006, 49, 5912-5931. [CrossRef] [PubMed]

41. Peele, K.A.; Durthi, C.P.; Srihansa, T.; Krupanidhi, S.; Ayyagari, V.S.; Babu, D.J.; Indira, M.; Reddy, A.R.; Venkateswarulu, T.C. Molecular docking and dynamic simulations for antiviral compounds against SARS-CoV-2: A computational study. Inform. Med. Unlocked 2020, 19, 100345. [CrossRef] [PubMed]

42. Hou, T.; Wang, J.; Zhang, W.; Xu, X. ADME evaluation in drug discovery. 6. Can oral bioavailability in humans be effectively predicted by simple molecular property-based rules? J. Chem. Inf. Model. 2007, 47, 460-463. [CrossRef] [PubMed]

43. Johnson, T.W.; Dress, K.R.; Edwards, M. Using the Golden Triangle to optimize clearance and oral absorption. Bioorg. Med. Chem. Lett. 2009, 19, 5560-5564. [CrossRef] 